\title{
Clinical Significance of Serum Kallistatin and ENOX1 Levels in Patients with Coronary Heart Disease
}

\author{
Rui Zhang Zhenjun Ji Junyan Cai Yongjun Li Genshan Ma \\ Department of Cardiology, Zhongda Hospital, School of Medicine, Southeast University, Nanjing, China
}

\section{Significance of the Study}

- Kallistatin and ENOX1 may participate in the regulation of oxidative stress and are thus potentially associated with atherosclerosis.

- This study showed that levels of kallistatin were decreased in patients with coronary heart disease (CHD), while levels of ENOX1 were increased in patients with acute coronary syndrome.

- Low levels of kallistatin or high levels of ENOX1 may lead to high Gensini score in CHD. Kallistatin may be of value in the diagnosis of CHD.

\section{Keywords}

Kallistatin · ENOX1 - Gensini · Biomarker · Coronary heart disease

\section{Abstract \\ Background: Kallistatin and ENOX1 are regulators of inflam- mation and oxidative stress which are typical pathological reactions in atherosclerosis. However, there is limited infor- mation of kallistatin and ENOX1 in coronary heart disease (CHD). Methods: Fifty healthy controls, 56 stable angina pec- toris (SAP) patients, and 47 acute coronary syndrome (ACS) patients were included in this study. Levels of kallistatin and ENOX1 in serum were measured by ELISA. $X^{2}$ test was per- formed to analyze categorical data. ANOVA, Pearson correla- tion analysis, and multiple linear regression were performed to analyze the numerical data. Finally, receiver operating characteristic $(\mathrm{ROC})$ curve was applied to assess the diagnos- tic value of kallistatin in CHD. Results: Among the 153 par-}

ticipants, $59.5 \%$ were male and the average age was $63.8 \pm$ 11.39 years. Compared with the control group, kallistatin expression was decreased in the SAP and ACS groups while expression of ENOX1 was increased in the ACS group ( $p<$ $0.05)$. Pearson correlation analysis showed that the kallistatin level was negatively correlated with the Gensini score $(r=$ $-0.210, p<0.01)$, white blood cell (WBC) count $(r=-0.283$, $p<0.001)$, and triglyceride levels $(r=-0.242, p<0.01)$ and positively correlated with age $(r=0.353, p<0.001)$ and highdensity lipoprotein cholesterol $(r=0.310, p<0.001)$. ENOX1 expression was positively correlated with WBC count $(r=$ $0.244, p<0.01)$, international normalized ratio $(r=0.177, p<$ $0.05)$, and Gensini score $(r=0.201, p<0.05)$. Multiple linear regression showed that $\mathrm{Cr}$, alanine transaminase, glucose, and kallistatin are independent predictors for Gensini score. The ROC curve showed that kallistatin had the highest diagnostic significance ( $p=0.007)$ when the area under curve was 0.636 , with a sensitivity of 0.735 and a specificity of 0.495 . Conclusion: Expression of kallistatin was decreased in karger@karger.com www.karger.com/mpp

Karger $\stackrel{\text { ' }}{5}$ BOPEN ACCESS
(C) 2020 The Author(s)

Published by S. Karger AG, Basel

This is an Open Access article licensed under the Creative Commons Attribution-NonCommercial-4.0 International License (CC BY-NC) (http://www.karger.com/Services/OpenAccessLicense), applicable to the online version of the article only. Usage and distribution for commercial purposes requires written permission.
Correspondence to:

Yongjun Li, liyongjunnj@ hotmail.com Genshan Ma, magenshan@ hotmail.com 
CHD patients and that of ENOX1 was increased in ACS patients. Kallistatin and ENOX1 were closely connected with the severity of CHD, and kallistatin may be helpful in the diagnosis of CHD.

(c) 2020 The Author(s)

Published by S. Karger AG, Basel

\section{Introduction}

Coronary heart disease (CHD) is one of the world's most common diseases with high fatality and disability rates [1]. CHD mainly consists of stable angina pectoris (SAP) and acute coronary syndrome (ACS) which is subcategorized into ST-elevation myocardial infarction, non-ST-elevation myocardial ischemia, and unstable angina $[2,3]$. Atherosclerosis is the most common pathological mechanism of CHD [4]. According to previous studies, activation of inflammatory response, the release of cytokines and chemokines, and enhancement of oxidative stress are all involved in atherosclerosis formation. Oxidative stress has been considered to be a core factor in the pathogenesis of atherosclerosis [5].

Kallistatin is a kallikrein-binding protein and widely distributed in various tissues and body fluids. As an endogenous serine proteinase inhibitor (serpin), kallistatin plays a vital role in a variety of diseases through regulation of inflammation, oxidative stress, apoptosis, and angiogenesis [6-8]. NADH oxidase, a plasma membrane electron transport complex, is a crucial source of reactive oxygen species and a key element in the activation of oxidative stress [9]. ENOX1 is a highly conserved NADH oxidase that oxidizes NADH to NAD+ [10]. Kallistatin was previously reported to inhibit superoxide formation and NADH oxidase activity [11]. Therefore, there may exist a biological connection between kallistatin and ENOX1.

Even though oxidative stress has been proven to predict the risk of cardiovascular events of CHD patients decades ago [12], the clinical roles of kallistatin and ENOX1 in CHD are yet to be explored. In this study, we examined the expression profile of kallistatin and ENOX1 in the serum of CHD patients and tried to explore the relationship between these 2 factors and CHD.

\section{Methods}

\section{Study Population}

The study was approved by the Ethics Committee of Southeast University affiliated to Zhongda Hospital, and all participants provided informed written consent before they were included in the study. A total of 153 patients hospitalized in the cardiovascular department from January 1 to March 28, 2017 were enrolled in our study. Patients suffering from more than one of the following diseases were excluded: chronic heart failure (grade III-IV), myocarditis, cardiomyopathy, pericardial disease, moderate to severe valve disease, severe hepatic or renal disease, thyroid disease, severe infection, systemic autoimmune diseases, and malignancy. All subjects were examined by coronary angiography to determine the extent of coronary artery lesions. Forty-seven patients of the enrolled patients were assigned to ACS group, which included unstable angina, non-ST-elevation myocardial ischemia, and ST-elevation myocardial infarction. The specific definition of ACS has been previously reported [13]. Fifty-six patients who had at least one major branch stenosis over 50\% without any indications of ACS were classified into the SAP group. The remaining 50 patients who had normal coronary arteries were assigned to the control group.

\section{Baseline Information Collection}

Baseline characteristics of subjects, including age, sex, history of hypertension, hyperlipidemia, diabetes, smoking, and drinking as well as family history of CHD were noted. The following parameters were studied: leukocyte count, red blood cell count, platelet count, creatine kinase-MB, myoglobin, international normalized ratio (INR), prothrombin time (PT), activated partial thromboplastin time (APTT), D-dimer, triglyceride (TG), total cholesterol, low-density lipoprotein cholesterol, high-density lipoprotein cholesterol (HDL-C), apolipoprotein A1, apolipoprotein B, lipoprotein a (lipoa), blood glucose, and $\mathrm{Cr}$ were collected. The left ventricular ejection fraction (LVEF), interventricular septum, left ventricular (LV), left ventricular posterior wall (LVPW), and left atrial (LA) were evaluated by echocardiography.

\section{Measurements of Kallistatin and ENOX1}

Blood samples from all the participants were collected prior to coronary angiography and stored in vacuum blood collection tubes containing inert separating gum for $15 \mathrm{~min}$ before serum separation. Blood samples were centrifuged at $1,000 \mathrm{~g}$ for $10 \mathrm{~min}$ at $4^{\circ} \mathrm{C}$, and serum was collected and cryopreserved at $-80^{\circ} \mathrm{C}$ until testing. The levels of kallistatin and ENOX1 were detected by an ELISA kit (Shanghai Westang Bio-tech Co., Ltd., Shanghai, China) according to the manufacturer's instructions. The highest and lowest points of the standard curve for kallistatin were 8,000 and 125, while for ENOX1 were 2,000 and 31.2.

\section{Coronary Angiography}

Coronary angiography was performed using the standard Judkins' technique. The analysis of angiographic results was performed by 2 experienced interventional cardiologists who did not know any clinical information of these patients. Gensini score was used to assess the severity of CHD. The scoring method has been previously described [14], and the higher the score, the worse the condition of coronary heart disease (CHD).

\section{Statistical Analysis}

Data processing was carried out with SPSS 22.0 and GraphPad prism 7. Continuous parametric variables were presented as mean \pm standard deviation (mean \pm SD). $\chi^{2}$ test was performed for the categorical data. Parametric variables in groups were compared by ANOVA. The connection between variables with kallistatin and 
Table 1. Basic characteristics of the subjects

\begin{tabular}{|c|c|c|c|c|}
\hline & Control $(n=50)$ & $\operatorname{SAP}(n=56)$ & $\operatorname{ACS}(n=47)$ & $p$ value \\
\hline Age, years & $62.26 \pm 11.36$ & $63.39 \pm 9.95$ & $65.94 \pm 12.86$ & 0.269 \\
\hline Male, $n, \%$ & 19,38 & $34,60.7$ & $38,80.9$ & $<0.001$ \\
\hline \multicolumn{5}{|l|}{ Medical history, $n, \%$} \\
\hline $\mathrm{AF}$ & 2,4 & $7,12.5$ & $5,10.6$ & 0.29 \\
\hline Smoking & 9,18 & $17,30.4$ & $32,68.1$ & $<0.001$ \\
\hline Diabetes & 6,12 & $16,28.6$ & $14,29.8$ & 0.049 \\
\hline Hypertension & 24,48 & $40,71.4$ & $28,59.6$ & $<0.01$ \\
\hline PCI History & 0,0 & $16,28.6$ & $4,8.5$ & $<0.001$ \\
\hline \multicolumn{5}{|l|}{ Biochemistry detection } \\
\hline $\mathrm{RBC}, \times 10^{12} / \mathrm{L}$ & $4.57 \pm 0.54$ & $4.55 \pm 0.52$ & $4.505 \pm 0.6$ & 0.827 \\
\hline WBC, $\times 10^{9} / \mathrm{L}$ & $6.44 \pm 2.08$ & $6.6 \pm 1.66$ & $10.05 \pm 3.9^{\mathrm{a}}$ & $<0.001$ \\
\hline Neutrophil, $\times 10^{9} / \mathrm{L}$ & $4.41 \pm 1.97$ & $4.34 \pm 1.74$ & $7.91 \pm 3.1^{\mathrm{a}}$ & $<0.001$ \\
\hline Monocyte, $\times 10^{9} / \mathrm{L}$ & $0.35 \pm 0.11$ & $0.39 \pm 0.15$ & $0.45 \pm 0.16^{\mathrm{a}}$ & 0.003 \\
\hline Lymphocyte, $\times 10^{9} / \mathrm{L}$ & $1.55 \pm 0.58$ & $1.74 \pm 0.63$ & $1.55 \pm 0.93$ & 0.315 \\
\hline Basophil, $\times 10^{9} / \mathrm{L}$ & $0.02 \pm 0.01$ & $0.02 \pm 0.01$ & $0.03 \pm 0.05$ & 0.304 \\
\hline Eosinophil, $\times 10^{9} / \mathrm{L}$ & $0.11 \pm 0.12$ & $0.1 \pm 0.08$ & $0.13 \pm 0.19$ & 0.691 \\
\hline PT, S & $11.42 \pm 0.69$ & $11.25 \pm 1.72$ & $15.39 \pm 12.28^{\mathrm{b}}$ & 0.005 \\
\hline APTT, S & $36.27 \pm 37.33$ & $32.07 \pm 13.65$ & $49.5 \pm 40.77^{\mathrm{b}}$ & 0.023 \\
\hline INR & $1.06 \pm 0.06$ & $1.22 \pm 1.37$ & $1.41 \pm 1.08$ & 0.247 \\
\hline D-dimer, $\mu \mathrm{g} / \mathrm{L}$ & $103.72 \pm 121.53$ & $103.71 \pm 143.52$ & $178.19 \pm 202.31$ & 0.031 \\
\hline $\mathrm{TC}, \mathrm{mmol} / \mathrm{L}$ & $4.59 \pm 1.04$ & $4.22 \pm 1.13$ & $4.43 \pm 0.81$ & 0.207 \\
\hline $\mathrm{TG}, \mathrm{mmol} / \mathrm{L}$ & $1.55 \pm 1.11$ & $1.79 \pm 2.47$ & $2.12 \pm 1.99$ & 0.392 \\
\hline $\mathrm{LDL}-\mathrm{C}, \mathrm{mmol} / \mathrm{L}$ & $2.72 \pm 0.81$ & $2.49 \pm 0.84$ & $2.8 \pm 0.61$ & 0.121 \\
\hline $\mathrm{HDL}-\mathrm{C}, \mathrm{mmol} / \mathrm{L}$ & $1.25 \pm 0.27$ & $1.13 \pm 0.26$ & $0.98 \pm 0.16$ & $<0.001$ \\
\hline ApoA1, g/L & $1.13 \pm 0.22$ & $2.57 \pm 11.09$ & $0.88 \pm 0.19$ & 0.425 \\
\hline ApoB, g/L & $0.82 \pm 0.21$ & $0.78 \pm 0.21$ & $0.84 \pm 0.17$ & 0.07 \\
\hline Lipoa, mg/L & $318.85 \pm 271.95$ & $327.52 \pm 372.52$ & $339.09 \pm 251.2$ & 0.955 \\
\hline Total protein, $g / \mathrm{L}$ & $65.61 \pm 12$ & $64.75 \pm 5.71$ & $61.3 \pm 5.92^{\mathrm{b}}$ & 0.032 \\
\hline Albumin, $g / L$ & $41.18 \pm 7.67$ & $41.65 \pm 3.98$ & $37.62 \pm 4.43^{\mathrm{a}}$ & 0.001 \\
\hline Prealbumin, g/L & $0.21 \pm 0.06$ & $0.21 \pm 0.05$ & $0.16 \pm 0.06$ & 0.000 \\
\hline $\mathrm{Cr}, \mathrm{g} / \mathrm{L}$ & $71.46 \pm 17.54$ & $79.86 \pm 24.58$ & $95.51 \pm 33.33^{\mathrm{a}}$ & $<0.001$ \\
\hline $\mathrm{BUN}, \mathrm{mmol} / \mathrm{L}$ & $5.22 \pm 1.31$ & $5.78 \pm 1.4$ & $6.78 \pm 2.74^{\mathrm{a}}$ & $<0.001$ \\
\hline $\mathrm{ALT}, \mathrm{U} / \mathrm{L}$ & $23.62 \pm 15.05$ & $26.72 \pm 27.09$ & $42.89 \pm 23.25^{\mathrm{a}}$ & $<0.001$ \\
\hline $\mathrm{AST}, \mathrm{U} / \mathrm{L}$ & $22.13 \pm 9.19$ & $23.5 \pm 12.81$ & $149.15 \pm 148.78^{a}$ & $<0.001$ \\
\hline Tbil, $\mu \mathrm{mol} / \mathrm{L}$ & $11.59 \pm 6.3$ & $11.73 \pm 9.93$ & $15.4 \pm 11.21$ & 0.093 \\
\hline $\mathrm{Dbil}, \mu \mathrm{mol} / \mathrm{L}$ & $2.66 \pm 1.98$ & $2.46 \pm 0.99$ & $4.95 \pm 8.26^{\mathrm{b}}$ & 0.024 \\
\hline Uric acid, $\mu \mathrm{mol} / \mathrm{L}$ & $319.23 \pm 95.62$ & $312.03 \pm 109.71$ & $373.28 \pm 100.74^{\mathrm{b}}$ & 0.007 \\
\hline Glucose, mg/L & $5.59 \pm 1.07^{\mathrm{c}}$ & $7.07 \pm 3.23$ & $7.94 \pm 4.14$ & 0.001 \\
\hline \multicolumn{5}{|l|}{ Echocardiography index } \\
\hline LVEF, \% & $0.67 \pm 0.06$ & $0.64 \pm 0.11$ & $0.61 \pm 0.12^{\mathrm{b}}$ & 0.039 \\
\hline IVS, $\mathrm{cm}$ & $0.98 \pm 0.14$ & $1.03 \pm 0.23$ & $1.08 \pm 0.13$ & 0.057 \\
\hline $\mathrm{LV}, \mathrm{cm}$ & $4.55 \pm 0.39$ & $4.68 \pm 0.51$ & $4.83 \pm 0.6$ & 0.089 \\
\hline LVPW, $\mathrm{cm}$ & $0.93 \pm 0.12$ & $0.99 \pm 0.15$ & $1.07 \pm 0.14^{\mathrm{a}}$ & $<0.001$ \\
\hline $\mathrm{LA}, \mathrm{cm}$ & $3.66 \pm 0.44$ & $3.91 \pm 0.62$ & $3.97 \pm 0.48$ & 0.024 \\
\hline
\end{tabular}

Data expressed as mean \pm SD or $\mathrm{n}(\%)$. SAP, stable angina pectoris; ACS, acute coronary syndrome; AF, atrial fibrillation; WBC, white blood cell; PT, prothrombin time; APTT, activated partial thromboplastin time; INR, international standardized ratio; TC, total cholesterol; TG, triglyceride; LDL-C, low-density lipoprotein cholesterol; HDL-C, high-density lipoprotein cholesterol; ApoA1, apolipoprotein A1; ApoB, apolipoprotein B; lipoa, lipoprotein a; ALT, alanine aminotransferase; AST, aspartate aminotransferase; Tbil, total bilirubin; Dbil, direct bilirubin; LVEF, left ventricular ejection fraction; IVS, interventricular septal; LV, left ventricle; LVPW, left ventricular posterior wall; LA, left atrial. ${ }^{\mathrm{a}} p<0.001$ in comparison with the control and SAP group. ${ }^{\mathrm{b}} p<0.05$ in comparison with the control and SAP group. ${ }^{c} p<0.05$ in comparison with the SAP and ACS group. 


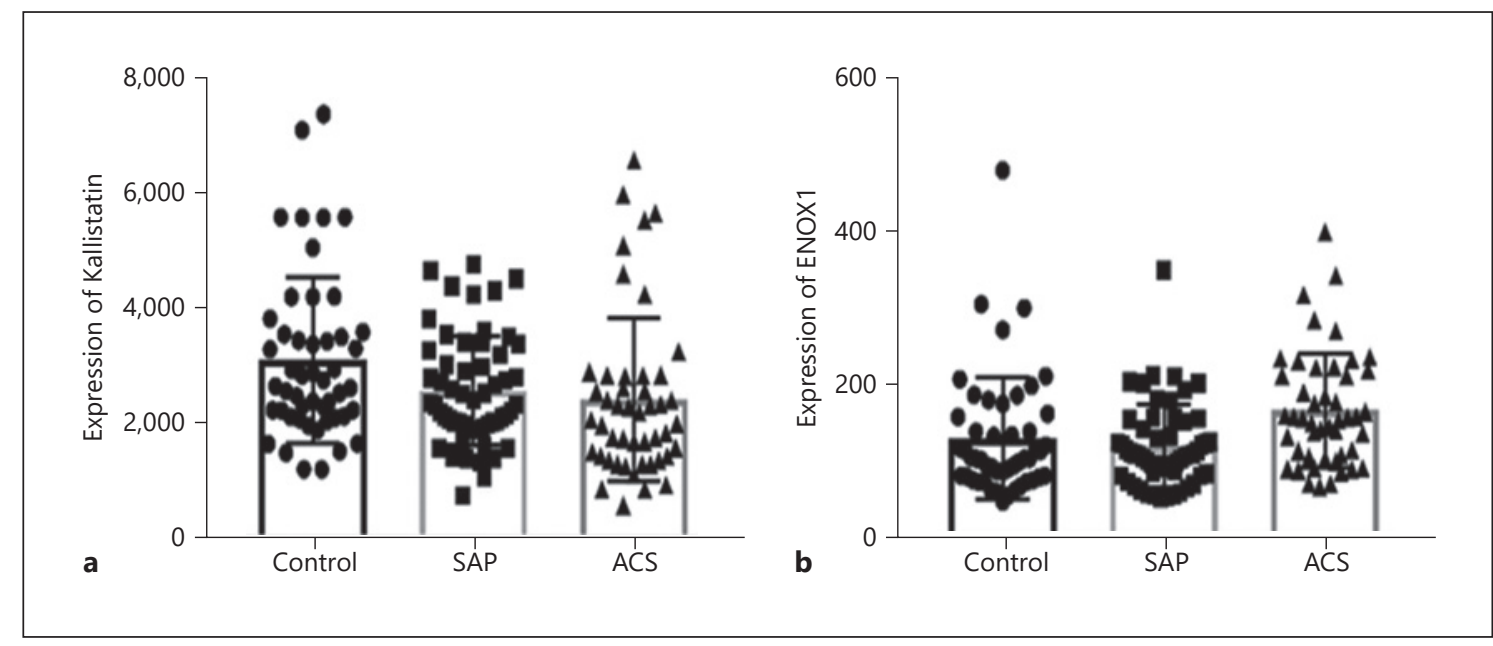

Fig. 1. ELISA levels of kallistatin (a) and ENOX1 (b) in controls, SAP, and ACS. SAP, stable angina pectoris; ACS, acute coronary syndrome.

Table 2. Comparisons of serum levels of kallistatin and ENOX1 in patients with CHD and control group

\begin{tabular}{lcccr}
\hline & Control $(n=50)$ & SAP $(n=56)$ & ACS $(n=47)$ & $p$ value \\
\hline Kallistatin, pg/mL & $3,094.69 \pm 1,435.65^{\mathrm{a}}$ & $2,564.15 \pm 955.32$ & $2,411.85 \pm 1,427.91$ & 0.024 \\
ENOX1, pg/mL & $129.23 \pm 79.43$ & $119.07 \pm 53.98$ & $166.53 \pm 74.49^{\mathrm{b}}$ & 0.002 \\
Gensini score & $1.44 \pm 2.91$ & $17.41 \pm 19.16^{\mathrm{c}}$ & $58.3 \pm 41.24^{\mathrm{d}}$ & $<0.001$ \\
\hline
\end{tabular}

Data expressed as mean \pm SD or $\mathrm{n}(\%)$. CHD, coronary heart disease; SAP, stable angina pectoris; ACS, acute coronary syndrome. ${ }^{\mathrm{a}} p<0.05$ in comparison with the control and SAP group. ${ }^{\mathrm{b}} p<0.05$ in comparison with the control and SAP group. ${ }^{c} p<0.001$ in comparison with the control group. ${ }^{\mathrm{d}} p<0.001$ in comparison with the control and SAP group.

ENOX1 was assessed using Pearson correlation analysis. Multiple linear regression analysis was used to determine the correlation between kallistatin and Gensini score with related factors. The specificity, sensitivity, and area under the curve of the receiver operating characteristic (ROC) curve were also computed to assess whether kallistatin could act as a biomarker for CHD patients. $p<$ 0.05 was considered to be significant for all tests.

\section{Results}

\section{Baseline Characteristics of Patients}

The general data of the study population are shown in Table 1. Among the 153 participants, there was no significant difference in age and atrial fibrillation history in the control, SAP, and ACS groups $(p>0.05)$. However, there were significant differences in sex, smoking, diabetes, hypertension, and PCI history $(p<0.05)$. Results of laboratory tests showed that there were no significant dif- ferences in the counts of RBC, lymphocytes, eosinophils, and basophils among 3 groups $(p>0.05)$. Compared with the control group and SAP group, white blood cells (WBCs), neutrophils, and monocytes in the ACS group were significantly increased $(p<0.01)$, as well as the value of PT, APTT, and DD dimers $(p<0.05)$. There were significant differences in HDL-C between all the groups $(p<0.001)$, and the highest level was in the control group. $\mathrm{Cr}, \mathrm{BUN}$, alanine aminotransferase (ALT), aspartate aminotransferase, total protein, albumin, direct bilirubin, and uric acid were all significantly different in the ACS group compared with the other 2 groups $(p<0.05)$. Compared with the control group, SAP and ACS groups had higher glucose $(p<0.01)$. Echocardiography showed there were significant differences in LVEF\%, LVPW, and LA. The ACS group showed the lowest LVEF\% $(p<0.05)$, the highest LVPW $(p<0.001)$, and the highest LA $(p<$ 0.05). 


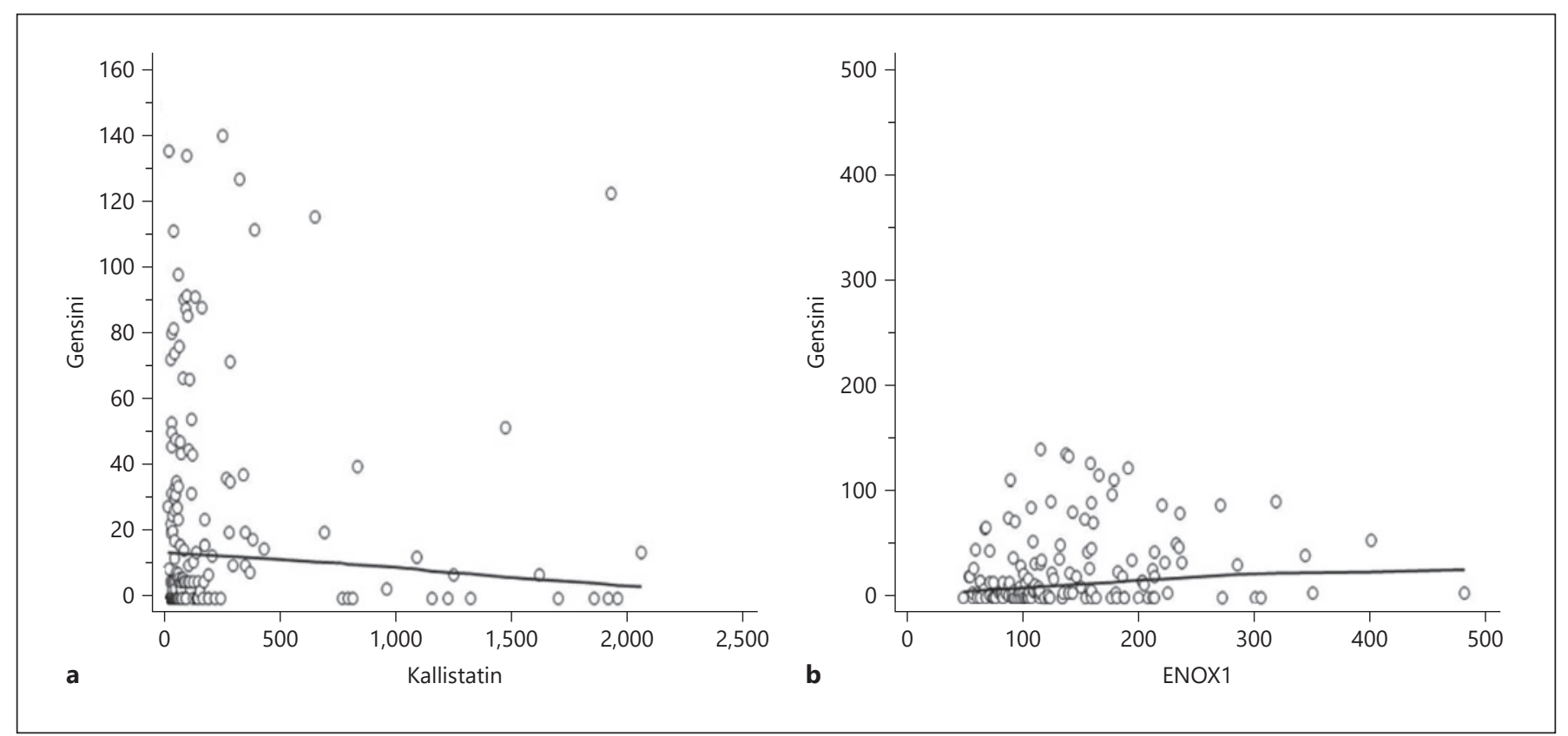

Fig. 2. Scatter (dot) plot with Pearson correlation analysis between kallistatin and Gensini score (a), as well as ENOX1 and Gensini score (b).

Table 3. Correlation analysis of circulating kallistatin and ENOX1 levels with cardiac risk factors

\begin{tabular}{lccccc}
\hline & \multicolumn{2}{l}{ Kallistatin } & & \multicolumn{2}{l}{ ENOX1 } \\
\cline { 2 - 3 } \cline { 6 - 6 } & $R$ & $p$ value & & $R$ & $p$ value \\
\hline Gensini score & -0.193 & 0.017 & & 0.178 & 0.028 \\
WBC & -0.262 & 0.001 & & 0.213 & 0.009 \\
TG & -0.224 & 0.008 & & 0.005 & 0.957 \\
Age & 0.360 & 0.000 & & 0.049 & 0.549 \\
HDL & 0.303 & 0.000 & & -0.126 & 0.143 \\
INR & -0.032 & 0.697 & & 0.163 & 0.048 \\
\hline
\end{tabular}

WBC, white blood cell; TG, triglyceride; INR, international normalized ratio.

\section{Comparison of Serum Levels of Kallistatin and}

ENOX1 in CHD and Control Group

Compared with the control group, kallistatin level was significantly decreased in the SAP group and ACS group $(3,094.69 \pm 1,435.65$ vs. $2,564.15 \pm 955.32$ vs. $2,411.85 \pm$ $1,427.91)$, and ENOX1 expression was significantly increased in ACS group (129.23 \pm 79.43 vs. $119.07 \pm 53.98$ vs. $166.53 \pm 74.49)(p<0.05)$ (Table 2; Fig. 1). There were significant differences in Gensini scores among all groups $(p<0.001)$ with the highest in the ACS group.

Value of Kallistatin and ENOX1 in CHD Patients

\section{Pearson Correlation Analysis of Kallistatin and ENOX1}

Pearson correlation analysis (Table 3 ) showed that kallistatin expression was negatively correlated with the Gensini score $(r=-0.193, p=0.017)$, WBC count $(r=$ $-0.262, p=0.001)$, and TG levels $(r=-0.224, p=0.008)$ and positively correlated with age $(r=0.360, p<0.001)$ and HDL-C $(r=0.303, p<0.001)$. ENOX1 expression was positively correlated with WBC count $(r=0.213, p=$ $0.009)$, INR $(r=0.163, p=0.048)$, and Gensini scores $(r=0.178, p=0.028)$. Scatter (dot) plot with Pearson correlation analysis between Kallistatin and Gensini score, as well as ENOX1 and Gensini score, were shown in Figure 2.

\section{Multiple Linear Regression of Kallistatin and Gensini Score}

Multiple linear regression showed that age, HDL-C, lipoa, and WBCs were the multiple factors influencing kallistatin level (Table 4). The fitting equation: [Kallistatin $]=41.364 \times$ age $+1,489.609 \times$ HDL-C- $0.832 \times$ lipoa-70.589 $\times$ WBC-775.579 $\left(R^{2}=0.308, F=12.444, p<\right.$ $0.001)$. Cr, ALT, glucose, and kallistatin are independently related to Gensini scores (Table 5); [Gensini] $=0.434 \times$ $\mathrm{Cr}+0.456 \times$ ALT- $0.005 \times$ Kallistatin $+1.798 \times$ glucose-22.496 $\left(R^{2}=0.46, F=7.061, p=0.009\right)$. 
Table 4. Multivariate linear regression analysis of kallistatin and related factors

\begin{tabular}{lrrrrrr}
\hline Factor & \multicolumn{1}{c}{ SD } & \multicolumn{1}{l}{ Beta } & \multicolumn{1}{l}{$t$} & \multicolumn{1}{l}{$95 \%$ CI } & $p$ value \\
\hline Age & 41.364 & 8.450 & 0.387 & 4.895 & $24.621-58.107$ & 0.000 \\
HDL & $1,489.609$ & 392.582 & 0.304 & 3.794 & $711.759-2,267.459$ & 0.000 \\
Lipoa & -0.832 & 0.318 & -0.208 & -2.617 & $-1.462 \sim-0.202$ & 0.010 \\
WBC & -70.589 & 35.173 & -0.161 & -2.007 & $-140.281 \sim-0.898$ & 0.047 \\
Constant & -775.579 & 802.302 & & -0.967 & $-2,365.238-814.079$ & 0.336 \\
\hline
\end{tabular}

Lipoa, lipoprotein a; WBC, white blood cell.

Table 5. Multivariate linear regression analysis of the Gensini score and related factors

\begin{tabular}{|c|c|c|c|c|c|c|}
\hline Factor & $B$ & SD & Beta & $t$ & $95 \%$ CI & $p$ value \\
\hline $\mathrm{Cr}$ & 0.434 & 0.092 & 0.346 & 4.734 & $0.253-0.616$ & 0.000 \\
\hline ALT & 0.456 & 0.109 & 0.307 & 4.192 & $0.241-0.671$ & 0.000 \\
\hline Kallistatin & -0.005 & 0.002 & -0.195 & -2.653 & $-0.009 \sim-0.001$ & 0.009 \\
\hline GLU & 1.798 & 0.766 & 0.170 & 2.346 & $0.282-3.313$ & 0.020 \\
\hline Constant & -22.496 & 10.866 & & -2.070 & $-43.985 \sim-1.006$ & 0.040 \\
\hline
\end{tabular}

ALT, alanine aminotransferase.

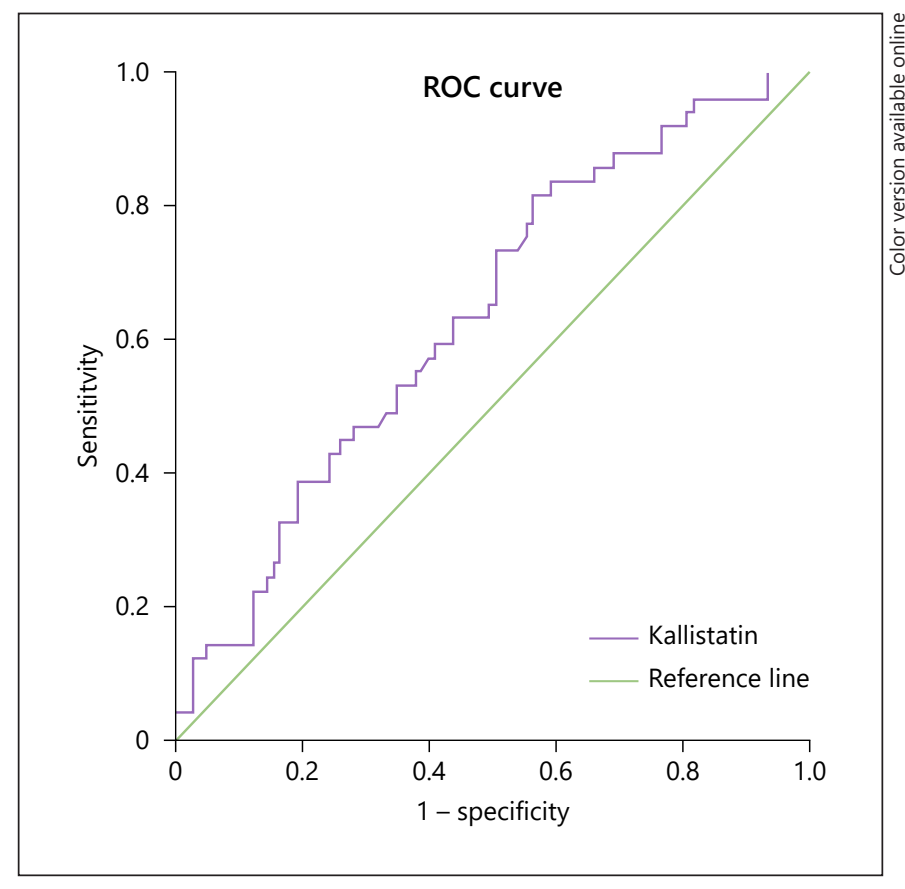

Fig. 3. The ROC curve of kallistatin analyzed with 50 control subjects and $103 \mathrm{CHD}$ patients. ROC, receiver operating characteristic; $\mathrm{CHD}$, coronary heart disease.

\section{ROC Curve of Kallistatin}

The ROC analysis was used to examine the role of kallistatin as a biomarker for CHD. The results showed that the area under the curve of kallistatin was 0.636 , with a sensitivity of 0.735 and a specificity of 0.495 (Fig. 3).

\section{Discussion}

CHD has become the most common disease and the leading cause of death around the world [15]. It is well known that atherosclerosis is the main pathological basis of CHD. Inflammation plays a primary role in the initial formation of atherosclerotic plaques and in the subsequent process of development into vulnerable plaques $[16,17]$. Once the vulnerable plaque in the coronary artery ruptures, thrombi form in the arteries leading to ischemic necrosis of the myocardium, resulting in a spectrum of clinical presentations referred to as ACS [18]. Oxidative stress, a phenomenon induced by the excessive accumulation of reactive oxygen species including superoxide radicals $\left(\mathrm{O}_{2}{ }^{--}\right)$, hydrogen peroxide, hydroxyl radicals $\left({ }^{\circ} \mathrm{OH}\right)$, and singlet oxygen $\left(1 \mathrm{O}_{2}\right)$ in cells, usually damages important cellular elements such as proteins and nucleic acids [19]. Oxidative stress can stimulate the inflammatory response by increasing the levels of ex- 
pression of proinflammatory cytokines [20]. Oxidative stress has been shown to be a major pathogenic factor in the progression of atherosclerosis [21]. Thus, research on oxidative stress and inflammation is of great significance in the diagnosis and treatment of atherosclerosis.

Kallistatin, an endogenous protein, could regulate a variety of cellular biological behaviors with its 2 special structural components: an active site and a heparinbinding domain. Kallistatin is widely expressed in multiple tissues and organs including heart and arterial vessels [22]. Recently, several researches revealed kallistatin to possess anti-inflammatory and anti-oxidative stress properties, thus inhibiting atherosclerotic plaque formation $[23,24]$. ENOX1, an NADH oxidase, is localized on the surface of the plasma membrane and catalyzes the oxidation of both $\mathrm{NADH}$ or hydroquinone, as well as protein disulfide-thiol interchange [25]. Lee et al. [26] found that the plasma membrane electron transport (containing ENOX1) pathway is activated by oxidized1-palmitoyl-2-arachidonyl-sn-glycerol-3-phosphocholine (ox-PAPC) and then induces oxidative stress and upregulates the expression of heme oxygenase-1 (HO-1) in human aortic endothelial cell; it is suggested that ENOX1 may be a regulator of oxidative stress in endothelial cells and a potential pathogenic factor in atherosclerosis.

In our study, expression of kallistatin was lower in the SAP group and the ACS group compared to the control group, which indicated a potential anti-atherosclerotic role of kallistatin. ENOX1 expression was significantly higher in the ACS group compared with control group and SAP group. Considering the association between oxidative stress and plaque rupture and subsequent complications of myocardial ischemic infarction [27, 28], we speculate that the elevation of ENOX1 in the ACS group is mainly due to increased oxidative stress caused by acute coronary events and may act as an independent risk factor for MI.

We also explored the correlations between kallistatin, ENOX1 and CHD-related factors that were assessed in our study, including leukocyte, RBC, platelet, CK-MB, Myo, INR, PT, APTT, D-dimer, TG, total cholesterol, low-density lipoprotein cholesterol, HDL-C, apolipoprotein $\mathrm{A} 1$, apolipoprotein $\mathrm{B}$, and blood glucose. We found that the expression of kallistatin was negatively correlated to WBC count and TG levels and is positively correlated to HDL-C, which further clarified that kallistatin may have a cardiovascular protective effect by regulating the inflammatory response and blood lipid level.

Value of Kallistatin and ENOX1 in CHD Patients
Next, we revealed a negative correlation between kallistatin and CHD Gensini scores, while ENOX1 was positively correlated with Gensini scores. At present, there is limited information about the mechanism of action of kallistatin and ENOX1 in atherosclerosis. First, kallistatin may increase the expression of endothelial protective factors such as nitrous oxide synthase, sirtuin 1, interleukin-10, superoxide dismutase 2 and thus reduce the size of plaques in the carotid artery [23]. In addition, kallistatin helps to reduce the accumulation of macrophages, the degree of oxidative stress and inflammatory response in the vascular wall $[23,24]$. In human aortic endothelial cells, ENOX1 could activate oxidative stress and has potential atherogenic effects [26]. In vivo experiments showed that anti-kallistatin promotes $\mathrm{NADH}$ oxidase activity and increases superoxide content in the heart [29]. Therefore, kallistatin may reduce oxidative stress partly by suppressing ENOX1, thus inhibiting the progression of atherosclerosis. Besides, kallistatin was positively correlated with age which is an independent risk factor for atherosclerosis [30]. Therefore, we speculate that the positive correlation between kallistatin and age may result from the compensatory mechanisms in CHD. Altogether, kallistatin and ENOX1 may interact with each other and influence the development of CHD.

Enhanced inflammatory response and oxidative stress are involved in CHD, especially in acute myocardial infarction [31, 32]. We have measured serum levels of kallistatin and ENOX1 in patients with different types of CHD. Dysregulated expressions of kallistatin and ENOX1 in CHD patients suggest that both of them may be related to atherosclerosis and may have potential value in predicting the severity of CHD. Although correlation analysis showed a negative correlation between kallistatin and Gensini score and a positive correlation between ENOX1 and Gensini score, multiple linear regression analysis further showed that kallistatin has significant predictive value for Gensini score, while ENOX1 does not. ROC analysis suggests that kallistatin may have diagnostic value in CHD.

\section{Conclusion}

Kallistatin was significantly decreased in patients with CHD (SAP and ACS), while ENOX1 was significantly increased in ACS. Kallistatin and ENOX1 are associated with the severity of CHD and kallistatin may serve as a novel biomarker for the diagnosis of CHD.

Med Princ Pract 2021;30:339-346

DOI: $10.1159 / 000510427$ 


\section{Acknowledgements}

We thank Dr. Abdlay for checking the manuscript for linguistic correctness. This study was funded by Jiangsu Provincial Key Medical Discipline (Laboratory ZDXKA2016023), Jiangsu Provincial Key Research and Development Program BE2016785 and the National Nature Science Foundation of China (No. 81600677).

\section{Statement of Ethics}

The study was approved by the Ethics Committee of Southeast University affiliated to Zhongda Hospital, and all participants provided informed written consent before they were included in the study.

\section{Conflict of Interest Statement}

The authors declare that they have no conflict of interest.

\section{References}

1 Torres N, Guevara-Cruz M, Velázquez-Villegas LA, Tovar AR. Nutrition and atherosclerosis. Arch Med Res. 2015 Jul;46(5):408-26.

2 Mehta SB, Wu WC. Management of coronary heart disease: stable angina, acute coronary syndrome, myocardial infarction. Prim Care. 2005 Dec;32(4):1057-81.

3 Singh A, Grossman SA. Coronary syndrome, acute. StatPearls. Treasure Island (FL): StatPearls Publishing StatPearls Publishing LLC 2018.

4 Golia E, Limongelli G, Natale F, Fimiani F, Maddaloni V, Pariggiano I, et al. Inflammation and cardiovascular disease: from pathogenesis to therapeutic target. Curr Atheroscler Rep. 2014 Sep;16(9):435.

5 Kattoor AJ, Pothineni NVK, Palagiri D, Mehta JL. Oxidative stress in atherosclerosis. Curr Atheroscler Rep. 2017 Sep 18;19(11):42.

6 Chao J, Bledsoe G, Chao L. Protective role of kallistatin in vascular and organ injury. Hypertension. 2016 Sep;68(3):533-41.

7 Chao J, Li P, Chao L. Kallistatin: doubleedged role in angiogenesis, apoptosis and oxidative stress. Biol Chem. 2017 Nov 27; 398(12):1309-17.

8 Frühbeck G, Gómez-Ambrosi J, Rodríguez A, Ramírez B, Valentí V, Moncada R, et al. Novel protective role of kallistatin in obesity by limiting adipose tissue low grade inflammation and oxidative stress. Metabolism. 2018 Oct; $87: 123-35$.

9 Weaver H, Shukla N, Ellinsworth D, Jeremy JY. Oxidative stress and vein graft failure: a focus on NADH oxidase, nitric oxide and eicosanoids. Curr Opin Pharmacol. 2012 Apr 12(2): $160-5$.

10 Venkateswaran A, Sekhar KR, Levic DS, Melville DB, Clark TA, Rybski WM, et al. The NADH oxidase ENOX1, a critical mediator of endothelial cell radiosensitization, is crucial for vascular development. Cancer Res. 2014 74(1):38-43.

11 Gao L, Yin H, S Smith R Jr, Chao L, Chao J. Role of kallistatin in prevention of cardiac remodeling after chronic myocardial infarction. Lab Invest. 2008 Nov;88(11):1157-66.

12 Heitzer T, Schlinzig T, Krohn K, Meinertz T, Münzel T. Endothelial dysfunction, oxidative stress, and risk of cardiovascular events in pa- tients with coronary artery disease. Circulation. 2001 Nov 27;104(22):2673-8.

13 Hahn J-Y, Song YB, Oh J-H, Cho D-K, Lee JB, Doh J-H, et al. 6-month versus 12 -month or longer dual antiplatelet therapy after percutaneous coronary intervention in patients with acute coronary syndrome (SMART-DATE): a randomised, open-label, non-inferiority trial. The Lancet. 2018 Mar 31;391(10127):127484.

$14 \mathrm{Yu}$ C, Chen M, Chen Z, Lu G. Predictive and prognostic value of admission neutrophil-tolymphocyte ratio in patients with CHD. Herz. 2016 Nov;41(7):605-13.

15 Kurd BJ, Dar MI, Shoaib M, Malik L, Aijaz Z, Asif I. Relationship between stress and coronary heart disease. Asian Cardiovasc Thorac Ann. 2014 Feb;22(2):142-7.

16 Libby P. Inflammation in atherosclerosis. Arterioscler Thromb Vasc Biol. 2012 Sep;32(9): 2045-51.

17 Hansson GK, Libby P, Tabas I. Inflammation and plaque vulnerability. J Intern Med. 2015 Nov;278(5):483-93.

18 Crea F, Libby P. Acute coronary syndromes: the way forward from mechanisms to precision treatment. Circulation. 2017 Sep 19; 136(12):1155-66

19 Pizzino G, Irrera N. Oxidative stress: harms and benefits for human health. Oxid Med Cell Longev. 2017;2017:8416763.

20 Hussain T, Tan B. Oxidative stress and inflammation: what polyphenols can do for us? Oxid Med Cell Longev. 2016;2016:7432797.

21 Förstermann U, Xia N, Li H. Roles of vascular oxidative stress and nitric oxide in the pathogenesis of atherosclerosis. Circ Res. $2017 \mathrm{Feb}$ 17;120(4):713-35.

22 Chao J, Guo Y, Li P, Chao L. Role of kallistatin treatment in aging and cancer by modulating miR-34a and miR-21 expression, Oxid Med Cell Longev. 2017;2017:5025610.

23 Yao Y, Li B, Liu C, Fu C, Li P, Guo Y, et al. Reduced plasma kallistatin is associated with the severity of coronary artery disease, and kallistatin treatment attenuates atherosclerotic plaque formation in mice. J Am Heart Assoc. 2018;7(21):e009562.

24 Li B, Sheng Z, Liu C, Qian L, Wu Y, Wu Y, et al. Kallistatin inhibits atherosclerotic inflam- mation by regulating macrophage polarization. Hum Gene Ther. 2019 Mar;30(3):33951.

25 Jiang Z, Gorenstein NM, Morré DM, Morre DJ. Molecular cloning and characterization of a candidate human growth-related and timekeeping constitutive cell surface hydroquinone (NADH) oxidase. Biochemistry. 2008 Dec 30;47(52):14028-38.

26 Lee S, Li R, Kim B, Palvolgyi R, Ho T, Yang QZ, et al. Ox-PAPC activation of PMET system increases expression of heme oxygenase-1 in human aortic endothelial cell. J Lipid Res. 2009 Feb;50(2):265-74.

27 Neri M, Fineschi V, Di Paolo M, Pomara C Riezzo I, Turillazzi E, et al. Cardiac oxidative stress and inflammatory cytokines response after myocardial infarction. Curr Vasc Pharmacol. 2015;13(1):26-36.

28 Mury P, Chirico EN, Mura M, Millon A, Canet-Soulas E, Pialoux V. Oxidative stress and inflammation, key targets of atherosclerotic plaque progression and vulnerability: potential impact of physical activity. Sports Med. 2018 Dec;48(12):2725-41.

29 Liu Y, Bledsoe G, Hagiwara M, Shen B, Chao L, Chao J. Depletion of endogenous kallistatin exacerbates renal and cardiovascular oxidative stress, inflammation, and organ remodeling. Am J Physiol Renal Physiol. 2012 Oct 15; 303(8):F1230-1238.

30 Wang JC, Bennett M. Aging and atherosclerosis: mechanisms, functional consequences, and potential therapeutics for cellular senescence. Circ Res. 2012 Jul 6;111(2):24559.

31 Li RJ, Ji WQ, Pang JJ, Wang JL, Chen YG, Zhang Y. Alpha-lipoic acid ameliorates oxidative stress by increasing aldehyde dehydrogenase- 2 activity in patients with acute coronary syndrome. Tohoku J Exp Med. 2013 Jan; 229(1):45-51.

32 Moreira DM, da Silva RL, Vieira JL, Fattah T, Lueneberg ME, Gottschall CA. Role of vascular inflammation in coronary artery disease potential of anti-inflammatory drugs in the prevention of atherothrombosis. Inflammation and anti-inflammatory drugs in coronary artery disease. Am J Cardiovasc Drugs. 2015 Feb;15(1):1-11. 